\title{
openheart Non-cardiac chest pain: prognosis and secondary healthcare utilisation
}

\author{
Karen Anne Mol, ${ }^{1}$ Agnieszka Smoczynska, ${ }^{2}$ Braim Mohammed Rahel, ${ }^{2}$ \\ Joan Gerard Meeder, ${ }^{2}$ Loes Janssen, ${ }^{3}$ Pieter A Doevendans, ${ }^{1}$ \\ Maarten-Jan Cramer ${ }^{1}$
}

\begin{abstract}
- Additional material is published online only. To view please visit the journal online (http://dx.doi.org/10.1136/10. 1136/openhrt-2018-000859).
\end{abstract}

To cite: Mol KA, Smoczynska A, Rahel BM, et al. Non-cardiac chest pain: prognosis and secondary healthcare utilisation Open Heart 2018;5:e000859. doi:10.1136/

openhrt-2018-000859

KAM and AS contributed equally.

Received 21 May 2018

Revised 9 July 2018

Accepted 12 July 2018

\section{Check for updates}

C Author(s) (or their employer(s)) 2018. Re-use permitted under CC BY-NC. No commercial re-use. See rights and permissions. Published by BMJ.

${ }^{1}$ Department of Cardiology, University Medical Centre, Utrecht, The Netherlands 2Department of Cardiology, VieCuri Medical Center, Venlo, The Netherlands

${ }^{3}$ Department of Clinical Epidemiology, VieCuri Medical Center, Venlo, The Netherlands

Correspondence to Dr Karen Anne Mol; k.a.mol@ umcutrecht.nl

\section{ABSTRACT}

Objective Presentations of non-cardiac chest pain (NCCP) to the emergency department (ED) are increasing. More knowledge of prognosis and healthcare utilisation of patients with NCCP is necessary to optimise their management.

Methods This study is a prospective, observational, prevalence-based cohort study conducted from September 2015 to February 2016 with 1-year follow-up including all patients 18 years and older referred to the ED with chest pain. Discharge diagnoses, mortality, major adverse cardiac events (MACE), re-presentations to the ED, hospitalisations, cardiac interventions and outpatient monitoring were assessed.

Results More than $60 \%$ of the 1239 patients presenting with chest pain were discharged with NCCP. The all-cause 1 -year mortality rate of patients with NCCP was $2.3 \%$ compared with $7.2 \%$ in patients with cardiac chest pain (CCP) $(p<0.001)$ and the occurrence of MACE was $5.1 \%$ vs $8.3 \%$, respectively $(p=0.026)$. Previous history of coronary artery disease (CAD) in patients with NCCP was identified as a predictive factor for MACE (OR $4.30(95 \% \mathrm{Cl} 1.24$ to 14.89$), p=0.021)$. Patients with NCCP had more noninvasive interventions than patients with CCP (proportion of 0.225 vs 0.165 per patient, $p<0.001$ ) and $13.7 \%$ of patients with NCCP re-presented at the ED within 1 year. Conclusion The majority of patients referred to the ED with chest pain are discharged with NCCP. The prognosis of patients with NCCP is better than patients with CCP; however, they are at risk for MACE due to a history of CAD. Patients with NCCP moreover use a substantial amount of medical resources, stressing the importance of good triage to minimise unnecessary healthcare utilisation while still preventing MACE.

\section{INTRODUCTION}

Non-cardiac chest pain (NCCP) has grown in clinical significance both in primary and secondary healthcare. There has been an increase in admissions with NCCP over the past decades, whereas the amount of presentations with acute coronary syndrome (ACS) has decreased. ${ }^{1}$ Population-based studies have estimated the prevalence of NCCP to range between $23 \%$ and $33 \%,{ }^{12}$ accounting for $2 \%-5 \%$ of all emergency presentations and more than $50 \%$ of all chest pain cases

\section{Key questions}

What is already known about this subject?

- More than $50 \%$ of all patients with chest pain presenting at the emergency department have non-cardiac chest pain (NCCP).

- Due to the heterogeneous nature of NCCP and its inherent diagnostic challenges, patients are frequently undergoing overinvestigation resulting in stress and high costs.

What does this study add?

- Our study compares the mortality, major adverse cardiac events (MACE) and diagnostic healthcare consumption of NCCP with patients with cardiac chest pain (CCP).

- We show that NCCP is more prevalent than CCP and despite the good nature of NCCP, the use of healthcare is substantial.

- Patients with NCCP do frequently have a history of coronary artery disease and a high presence of cardiovascular risk factors.

How might this impact on clinical practice?

- Triage during the first presentation with chest pain should be improved to minimise unnecessary hospital admissions and the consequential healthcare utilisation of patients with NCCP while still preventing MACE.

presenting at the emergency department (ED). ${ }^{13}$ Due to the heterogeneous nature of the condition, diagnosing the underlying cause of NCCP is challenging. ${ }^{45}$ NCCP is defined as chest pain not attributed to underlying ischaemic heart disease and may be of gastrointestinal, musculoskeletal, respiratory or psychological origin. ${ }^{1346}$ Moreover, there may be an overlap in the underlying conditions causing NCCP. ${ }^{1}$ As a consequence of these diagnostic challenges, the cost of NCCP has increased tremendously. ${ }^{1}$ In addition, there is a considerable persistence of symptoms resulting in continued utilisation of medical resources, with signs of overinvestigation in every 10th patient with persisting chest pain, despite the good prognosis. ${ }^{17}$ 
The primary aim of this study is to gain insight into the prognosis of patients with NCCP presenting at the ED compared with patients with cardiac chest pain (CCP), in terms of mortality and occurrence of major adverse cardiac events (MACE). Second, the study aims to identify baseline variables in patients with NCCP that increase the risk for developing a MACE. Finally, it provides an overview of the utilisation of secondary healthcare at the cardiology department by patients with chest pain. These insights will contribute to the optimal management of patients with NCCP.

\section{METHODS}

\section{Study design}

This study is a prospective, observational, prevalence-based cohort study conducted from the 1 September 2015 to 28 February 2016 with a year follow-up until the 28 February 2017.

\section{Study sample and setting}

All patients 18 years or older with chest pain who were referred to the ED suspected of ACS were included in this study. This included all patients with non-traumatic chest pain and other symptoms that can rise the suspicion of ACS. This study investigated patients referred from outside the hospital and thus excluded patients referred by specialists. The ED is located at VieCuri Medical Centre, a medium-sized teaching hospital and an off-site percutaneous coronary intervention (PCI) centre in the Netherlands.

\section{Data collection}

Data were collected by the attending physician and retrieved through medical files. A questionnaire was given to patients with additional questions not readily available in the medical files to minimise missing data. Collected data included demographics, history, symptoms, medication, discharge diagnosis made by the attending physician, mortality, occurrence of MACE, re-presentations at the ED, hospitalisations and cardiac interventions.

Ethical approval was waived by Medical Ethical Review Commission Utrecht, protocol number 15/382.

\section{Data analysis}

Statistical analyses were performed using IBM SPSS V.24. Descriptive statistics are summarised as proportions for categorical variables and mean with SD for continuous variables. Comparison between patients with NCCP and CCP was performed using the Students-t-test, Mann Whitney $U$ test or $\chi^{2}$ test, as appropriate. Possible factors in the NCCP subset related to the occurrence of MACE were investigated using logistic regression analysis. Factors with a p-value $<0.10$ in univariate models were considered for further investigation by means of a multivariate model and expressed as ORs and 95\% CIs. $\mathrm{P}<0.05$ was considered to be statistically significant. Patients with missing values were included, with exception of their missing values.

\section{Definitions}

Patients with CCP included patients with ACS and non-ischaemic heart disease. ACS was subdivided as ST-elevation myocardial infarction (STEMI), non-STEMI (NSTEMI) or unstable angina pectoris (UAP) ${ }^{8}$ Non-ischaemic heart disease included (myo-) pericarditis, heart rhythm problems, valvular disease and cardiomyopathies.

NCCP was defined as chest pain not attributed to underlying (ischaemic) heart disease and subdivided in NCCP of known origin (NCCP-K) and NCCP of unknown origin (NCCP-U). NCCP-K comprised patients who were discharged from the index admission with a non-cardiac diagnosis for their chest pain. This group included severe conditions such as pneumonia, pulmonary embolism, pneumothorax, aortic dissection and severe gastrointestinal disorders and also benign conditions such as gastro-oesophageal reflux disease, musculoskeletal disorders and psychological disorders. NCCP-U are patients who were discharged with chest pain not otherwise specified.

MACE included any STEMI, NSTEMI, UAP, PCI and coronary artery bypass grafting (CABG) after discharge. An ACS diagnosis and its corresponding treatment at initial admission was not considered a MACE. ${ }^{8}$

\section{RESULTS}

During the study period, 1322 patients, with a total of 1469 presentations, were referred to the ED with suspected ACS. Patients referred by specialists $(n=66)$ and patients who were referred with an out-of-hospital cardiac arrest $(n=17)$ were excluded from further analysis. There were 25 patients lost to follow-up due to them not living in the Netherlands or due to an unknown general practitioner (GP). GPs referred $1001(80.8 \%)$ patients and the remaining $238(19.2 \%)$ patients were referred by emergency medical transport or were self-referrals. CCP was diagnosed in $490(39.5 \%)$ patients and NCCP in 749 $(60.5 \%)$ patients. Further differentiations are shown in figure 1 .

\section{Baseline characteristics}

Characteristics at baseline differed between the patients with NCCP and CCP/ACS in mean age (60.6 vs $66.1 / 66.6$ years, $\mathrm{p}<0.001 / \mathrm{p}<0.001)$, male gender $(45.3 \%$ vs $66.5 / 66.8 \%, \mathrm{p}<0.001 / \mathrm{p}<0.001)$ and history of hypercholesterolemia $(39.3 \%$ vs $47.6 / 49.1 \%$, $\mathrm{p}=0.004$ / $\mathrm{p}=0.003$ ). Baseline differences between patients with NCCP and CCP (but not patients with ACS) were seen for cardiovascular disease (CVD) (42.9\% vs 48.6 , $\mathrm{p}=0.048)$ and coronary artery disease (CAD) $(29.1 \%$ vs $35.8 \%, \mathrm{p}=0.014$ ) (online supplementary table 1 ). The NCCP-K and NCCP-U subsets did not differ in baseline characteristics, with the exception of history of CVD $(37.4 \%$ vs $47.8 \%, \mathrm{p}=0.004)$ and CAD $(23.9 \%$ vs $33.9 \%, \mathrm{p}=0.003)$, which were both more prevalent in the NCCP-U subset. 


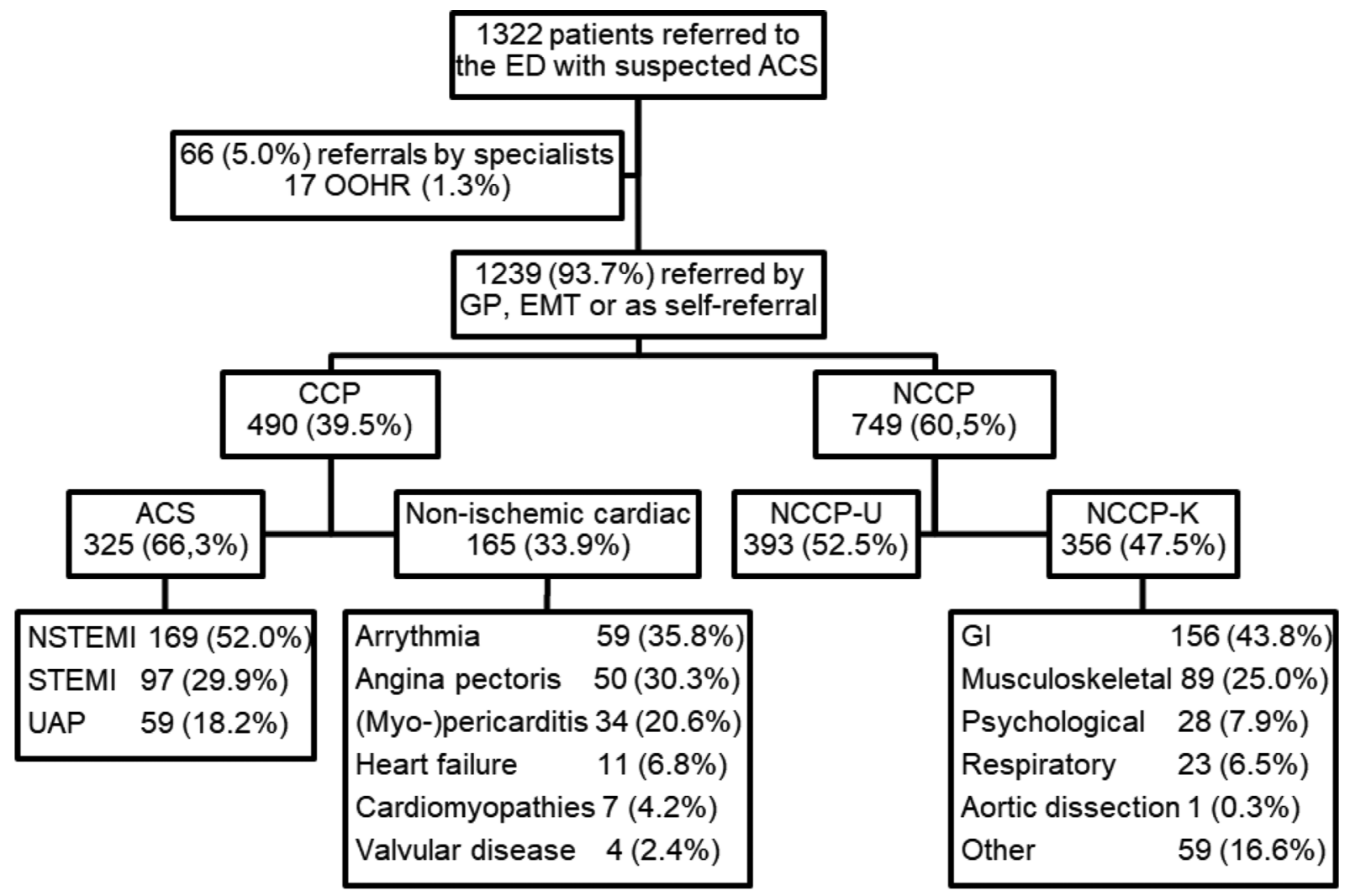

Figure 1 Study flow chart and diagnosis at discharge from ED. ACS, acute coronary syndrome; CCP, cardiac chest pain; ED, emergency department; EMT, emergency medical transport; GI: gastrointestinal disorders; GP, general practitioner, NCCP, non-CCP; NCCP-K, NCCP of known origin; NCCP-U, NCCP of unknown origin; NSTEMI, non-STEMI; STEMI, ST-elevated myocardial infarction; UAP, unstable angina pectoris.

\section{Changes in discharge diagnosis during 1-year follow-up}

A number of the initial diagnoses were revised within the 1-year follow-up. In the patients with ACS, all STEMI and NSTEMI diagnoses remained unchanged, whereas $74.6 \%$ of the UAP diagnoses remained the same. In the latter group, $9(15.2 \%)$ patients received a diagnosis of non-ischaemic heart disease and $6(10.2 \%)$ diagnoses became NCCP-K. In the group of patients with chest pain caused by non-ischaemic heart disease, $145(88.0 \%)$ diagnoses remained unchanged and the remaining cases were adjusted into ACS (0.6\%), NCCP-K (1.2\%) and NCCP-U $(10.2 \%)$. In the NCCP-K group, $6(1.7 \%)$ diagnoses were adjusted into non-ischaemic heart disease and 136 (38.2\%) diagnoses into NCCP-U, when all other diagnoses were excluded. The remaining 215 (60.4\%) diagnoses remained NCCP-K.

The majority of the NCCP-U diagnoses, namely 336 $(85.5 \%)$, remained unchanged. Of the remaining patients, $2(0.5 \%)$ diagnoses were adjusted into UAP, 31 (7.9\%) into non-ischaemic heart disease and 24 (6.1\%) into NCCP-K.

\section{Prognosis}

A total of 52 patients died during the follow-up of 1 year. Kaplan-Meier survival plots are presented in figure 2 and mortality rates per group are summarised in table 1 .
Patients with CCP had a 1-year mortality rate of $7.2 \%$ and patients with ACS of $8.1 \%$, both higher compared with the NCCP group with a 1-year mortality rate of $2.3 \%(p<0.001)$.

The occurrence of MACE during follow-up was higher in the CCP and ACS groups compared with the NCCP group ( $8.3 \%$ and $8.7 \%$ vs $5.1 \%$, respectively, $\mathrm{p}=0.026)$ as portrayed in table 2. There was no difference between NCCP-K and NCCP-U subsets in the occurrence of MACE, except for the incidence of UAP which was more frequent in the NCCP-U subset $(0.0 \%$ vs $1.3 \%, \mathrm{p}=0.04)$. We also analysed the effect of a known history of CAD in the NCCP-U subset. The most striking difference was found in the occurrence of MACE ( $15.3 \%$ with known $\mathrm{CAD}$ vs $1.6 \%$ without, $\mathrm{p}<0.001$ ). This was also seen when comparing patient with known CVD and without CVD (occurrence of MACE in $10.8 \%$ vs $2.0 \%$, $\mathrm{p}<0.001)$. The mortality did not differ in patients with NCCP-U with or without CAD or CVD.

Possible predictors for the occurrence of MACE in the NCCP group were analysed. After correction for possible confounders, we identified one predictor, namely a history of CAD (OR 4.30 (95\% CI 1.24 to 14.89), $\mathrm{p}=0.021$ ) as shown in online supplementary table 2.

\section{Utilisation of secondary healthcare in the cardiology department}

Table 3 shows the utilisation of secondary healthcare in the cardiology department during the 1-year follow-up 

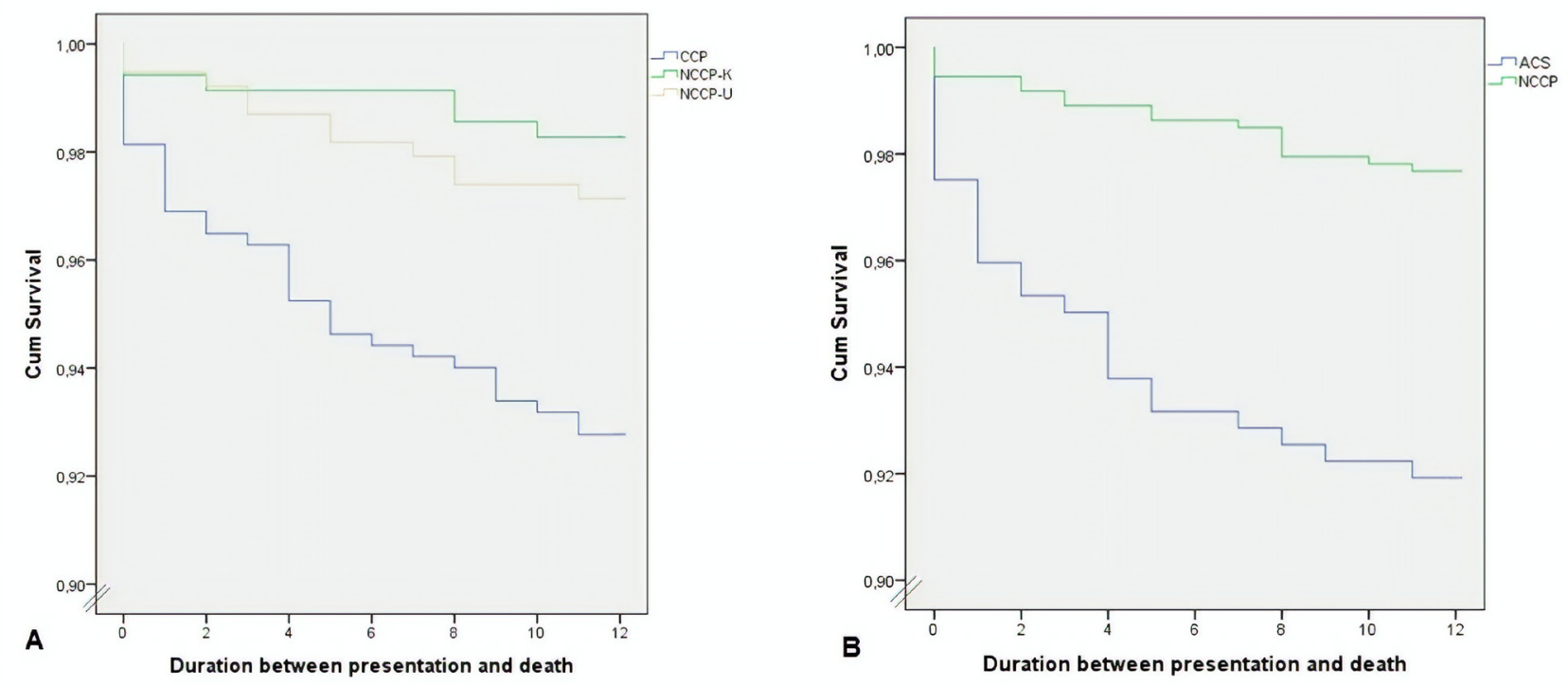

Figure 2 (A) Survival in CCP group compared to non-CCP (NCCP) of known (K) and unknown (U) origin; CCP vs NCCP, $\mathrm{p}<0.001$ (log-rank test); NCCP-K vs NCCP-U p=0.41 (log-rank test). (B) Survival in the ACS group compared to the NCCP group; $p<0.001$ (log-rank test). ACS, acute coronary syndrome; CCP, cardiac chest pain.

after discharge from the index admission. Patients with CCP had a higher utilisation of medical resources compared with patients with NCCP. The duration of rehospitalisation of patients with NCCP at the cardiology department was however similar to that of patients with CCP and a proportion of $13.7 \%$ of patients with NCCP re-presented at the ED.

Patients of the NCCP-U subset had a longer duration of hospitalisation, more re-presentations at the ED, more rehospitalisation, more outpatient department visits, more consultations by phone and a longer outpatient department monitoring than the NCCP-K subset.

\section{Cardiac interventions}

We analysed the amount of interventions performed within the patient groups (online supplementary table 3 ). We present proportions as some patients have undergone more than one intervention within the follow-up period. Patients with CCP and in particular patients with ACS underwent more PCIs or CABG (0.598/0.862 vs $0.047, \mathrm{p}<0.001 / \mathrm{p}<0.001)$ and more coronary angiography $(\mathrm{CAG})$ without intervention $(0.251 / 0.218$ vs 0.119 , $\mathrm{p}<0.001 / \mathrm{p}<0.001)$ than patients with NCCP. Patients with NCCP and in particular patients with NCCP-U underwent more non-invasive interventions such as CT-scans, SPECT or MRI's (0.165 in CCP group vs 0.290 in NCCP group, $\mathrm{p}<0.001$ and 0.349 in NCCP-U group, $\mathrm{p}<0.001$ ). The NCCP group was the group with the most patients without any intervention (24.7\% in CCP group, $39.5 \%$ in NCCP group, $\mathrm{p}<0.001)$.

\section{DISCUSSION}

\section{Prognosis}

The majority of the patients $(60.5 \%)$ referred to the ED with chest pain are discharged with NCCP. The prognosis of patients with NCCP is better in terms of survival (1-year mortality of $2.3 \%$ vs $7.2 \%$ and $8.1 \%$, respectively,

Table 1 Mortality rate

\begin{tabular}{|c|c|c|c|c|c|c|c|c|}
\hline & \multicolumn{5}{|c|}{ Diagnosis at discharge } & \multicolumn{3}{|l|}{$P$ values } \\
\hline & $\begin{array}{l}\text { CCP* }^{*} \\
(n=490)\end{array}$ & $\begin{array}{l}\text { ACS } \\
(n=325)\end{array}$ & $\begin{array}{l}\text { NCCP† } \\
(n=749)\end{array}$ & $\begin{array}{l}\text { NCCP-K } \\
(n=356)\end{array}$ & $\begin{array}{l}\text { NCCP-U } \\
(n=393)\end{array}$ & $\begin{array}{l}\text { CCP vs } \\
\text { NCCP }\end{array}$ & $\begin{array}{l}\text { ACS vs } \\
\text { NCCP }\end{array}$ & $\begin{array}{l}\text { NCCP-K vs } \\
\text { NCCP-U }\end{array}$ \\
\hline In-hospital mortality rate, $\mathrm{n}(\%)$ & $10(2.0)$ & $9(2.8)$ & $4(0.5)$ & $2(0.6)$ & $2(0.5)$ & 0.014 & 0.002 & 0.921 \\
\hline 6-month mortality rate, n (\%)‡ & $27(5.6)$ & $22(6.8)$ & $10(1.4)$ & $3(0.9)$ & $7(1.8)$ & $<0.001$ & $<0.001$ & 0.263 \\
\hline 1-year mortality rate, $\mathrm{n}(\%) \ddagger$ & $35(7.2)$ & $26(8.1)$ & $17(2.3)$ & $6(1.7)$ & $11(2.9)$ & $<0.001$ & $<0.001$ & 0.306 \\
\hline
\end{tabular}

${ }^{*} \mathrm{CCP}$ group includes patients with ACS.

†NCCP group is a sum of the patients of the NCCP-K and NCCP-U groups.

$\ddagger 7,6,9$ and 9 patients loss of follow-up in the CCP, ACS, NCCP-K and NCCP-U groups, respectively.

ACS, acute coronary syndrome; CCP, cardiac chest pain; NCCP, non-CCP; NCCP-K, NCCP of known origin; NCCP-U, NCCP of unknown origin. 
Table 2 Major adverse cardiac events during 1-year follow-up

\begin{tabular}{|c|c|c|c|c|c|c|c|c|}
\hline & \multicolumn{5}{|c|}{ Diagnosis at discharge } & \multicolumn{3}{|l|}{ P values } \\
\hline & $\begin{array}{l}\text { CCP* } \\
(N=471) \dagger\end{array}$ & $\begin{array}{l}\text { ACS } \\
(\mathrm{N}=310) \dagger\end{array}$ & $\begin{array}{l}\text { NCCP } \\
(N=729) \dagger\end{array}$ & $\begin{array}{l}\text { NCCP-K } \\
(N=345) \dagger\end{array}$ & $\begin{array}{l}\text { NCCP-U } \\
(\mathrm{N}=383) \dagger\end{array}$ & CCP vs NCCP & ACS vs NCCP & $\begin{array}{l}\text { NCCP-K vs } \\
\text { NCCP-U }\end{array}$ \\
\hline MACE, $n(\%)$ & $39(8.3)$ & $27(8.7)$ & $37(5.1)$ & $13(3.8)$ & $24(6.3)$ & 0.026 & 0.026 & 0.123 \\
\hline STEMI, n (\%) & $15(3.2)$ & $12(3.9)$ & $19(2.6)$ & $8(2.3)$ & $11(2.9)$ & 0.555 & 0.273 & 0.636 \\
\hline NSTEMI, n (\%) & $15(3.2)$ & $8(2.6)$ & $12(1.6)$ & $5(1.4)$ & $7(1.8)$ & 0.079 & 0.316 & 0.685 \\
\hline UAP, n (\%) & $8(1.7)$ & $6(1.9)$ & $5(0.7)$ & $0(0)$ & $5(1.3)$ & 0.098 & 0.072 & 0.033 \\
\hline CABG or PCI, $n(\%)$ & $12(2.5)$ & $9(2.9)$ & $16(2.2)$ & $7(2.0)$ & $9(2.3)$ & 0.692 & 0.495 & 0.764 \\
\hline
\end{tabular}

${ }^{*}$ CCP group includes patients with ACS.

†Exclusion of 19, 15, 20, 10 and 10 patients from analysis in the CCP, ACS, NCCP, NCCP-K and NCCP-U groups, respectively; in total 25 due to loss-to-follow-up and 14 due to in-hospital death.

‡NCCP group is a sum of patients with NCCP-K and NCCP-U.

ACS, acute coronary syndrome; MACE, Major adverse cardiac events defined as a combinations of the following: CABG, coronary artery bypass graft; CCP, cardiac chest pain; NCCP, non-CCP; NCCP-K, NCCP of known origin; NCCP-U, non-cardiac chest pain group of unknown origin; NSTEMI, non-STEMI; PCI, percutaneous coronary intervention; STEMI, ST-elevation myocardial infarction; UAP, unstable angina pectoris.

$\mathrm{p}<0.001)$ and occurrence of MACE $(5.1 \%$ vs $8.3 \%$ and $8.7 \%$, respectively, $\mathrm{p}=0.026$ ) compared with CCP and ACS groups. Previous studies showed similar results with 1-year mortality rates ranging from $1.4 \%$ to $4.2 \%$ for patients with NCCP and $14 \%$ to $19.9 \%$ for patients with ACS. ${ }^{4}$ Our reported 1-year mortality rate in the ACS group was lower than earlier studies, which may be related to the introduction of new sensitive markers combined with the more sensitive assays for troponins allowing more accurate diagnosis and earlier treatment of ACS. ${ }^{4}$ The mortality of the NCCP, of known and unknown origin, patients is comparable to the mortality of the general population; however, the occurrence of MACE in the NCCP, and in particular the patients with NCCP-U is considerably high, namely $5.1 \%$ and $6.3 \%$, respectively, in 1 year. Moreover, despite the lower occurrence of MACE in the NCCP group, the amount of STEMIs, NSTEMIs, UAP and even PCIs or CABG was not statistically significantly lower compared with the CCP group, though this could be due to the low incidence. The relatively high MACE in patients with NCCP may be a consequence of the overlapping cardiovascular risk factors at baseline in the NCCP and CCP groups. A history of CAD was identified as a predictive factor for the occurrence of MACE in the patients with NCCP. We also found that patients with NCCP-U with a history of CAD (or CVD) had a higher risk of developing a MACE in the follow-up. This is in line with previously reported findings by Ruddox $e t a l,{ }^{4}$ where it was stated that the prognosis of patients with NCCP is not necessarily benign due to the pre-existing CAD in approximately $40 \%$ of these patients. These findings suggest that patients with chest pain and a history of CAD should be monitored closely and might benefit more from a follow-up to prevent frequent re-presentations, re-hospitalisations and MACE.

\section{Consultations and interventions}

Patients with NCCP did not exceed the amount of visits for medical care compared with patients with ischaemic heart disease. In the organisation of the Dutch healthcare system GPs also play an important role in the management of NCCP and thereby decrease the healthcare utilisation in secondary healthcare. Healthcare utilisation in the primary healthcare setting was not included in this study.

Patients with NCCP, in particular the patients with NCCP-U, do use a substantial amount of medical resources at the cardiology department. The mean amount of re-presentations at the ED, duration of outpatient department monitoring and visits to the cardiologist of patients with NCCP-U exceeds that of patients with NCCP-K. Moreover, the amount of cardiac interventions including PCI, CABG, CAG and non-invasive imaging are also higher in the NCCP-U vs the NCCP-K group. It is believed that a lack of explanation for their symptoms may lead to psychological distress which drives patients with NCCP-U to seek medical advice, ${ }^{3}$ whereas patients who are given a specific diagnosis for their chest pain use less healthcare. $^{6}$ The higher amount of PCI in the NCCP group may be due to incidental findings of coronary artery stenosis that is not related to the chest pain complaints. In the process, patients with NCCP are exposed to risk and stress by unnecessary referral or admission to the hospital and the consequential interventions, posing a psychological burden on them as well as their families. ${ }^{9}{ }^{10}$ In order to combat the issues of overcrowding at the ED, the costs of healthcare and the exposure of patients to unnecessary hospital admissions and diagnostics, it is important to improve triage and follow-up of patients with chest pain. ${ }^{9}$

\section{Strengths and limitations}

All patients 18 years and older with chest pain referred from primary healthcare were included in this study, making it an unselected study population with a good representation of daily practice at the ED. However, this may result in a rather heterogeneous study population.

Furthermore, the study was a single-centre study and set at the ED, making it vulnerable for selection bias, with 


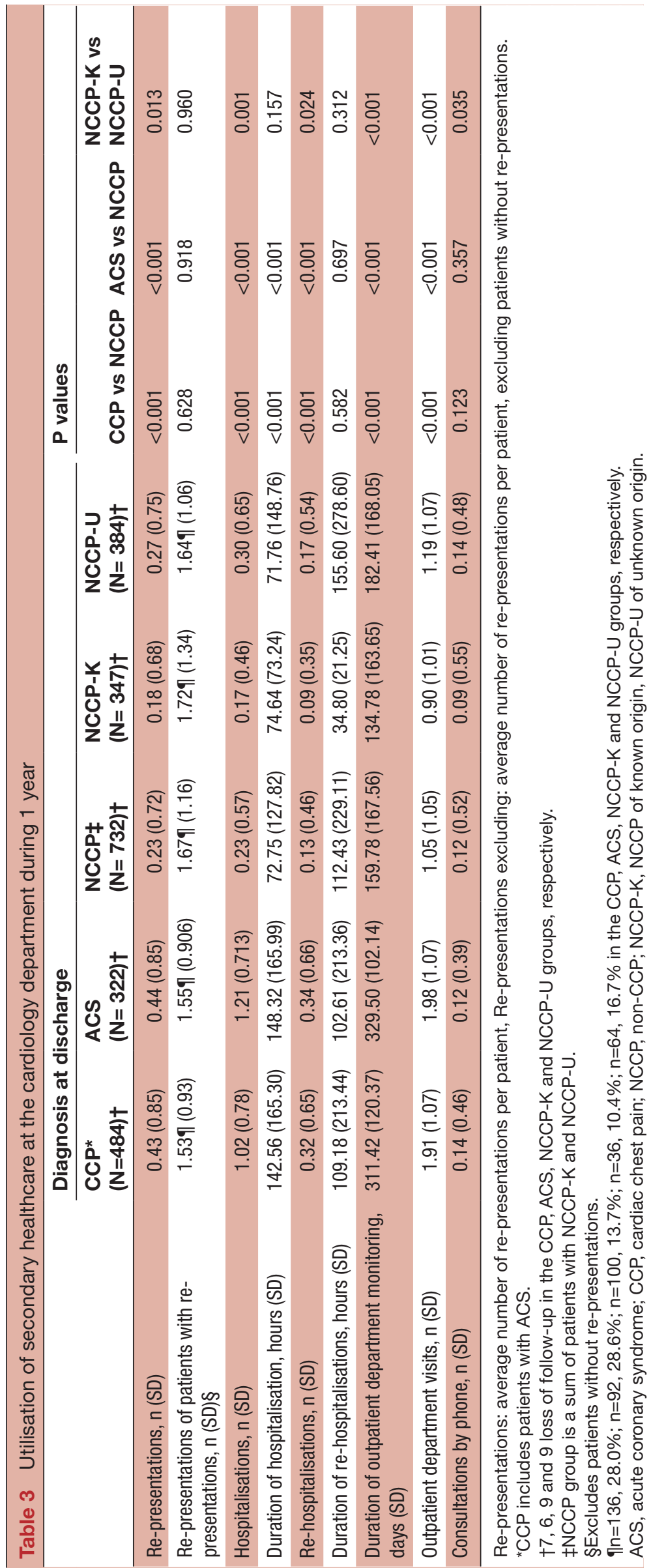


patients mostly referred after contact with a GP. In the Netherlands, GPs have a gatekeeper role and patients are advised to initially consult their GP for most symptoms. Therefore, the patients who are referred to the ED are more likely to have cardiovascular risk factors and prior history of CAD. As a consequence, our study population may have had a higher occurrence of MACE than the general population and patients in primary healthcare and even than in countries without GPs as gate keepers.

This single-centre study did include all patients referred from the region. A selection bias through expertise of the centre was not a factor.

\section{Conclusion and recommendations}

The majority of patients referred to the ED with chest pain are discharged with a non-cardiac diagnosis. The prognosis in patients with NCCP is better than patients with CCP; however, they are nevertheless at risk for the occurrence of MACE due to the presence of cardiovascular risk factors and prior history of CAD at baseline. NCCP, and in particular NCCP-U, patients moreover use a substantial amount of medical resources. Triage during the first presentation with chest pain should be improved to minimise unnecessary hospital admissions and the consequential healthcare utilisation of patients with NCCP while still preventing MACE.

Contributors KAM, AS: planning, conduct and report of the work, responsible for the overall content. BMR: planning and review of the work JGM: planning an review of the work. LJ: statistical support and review of the work. PAD: review of the work. MJMC: planning and review of the work.

Funding The authors have not declared a specific grant for this research from any funding agency in the public, commercial or not-for-profit sectors.

Competing interests None declared.

Patient consent Not required.

Ethics approval METC UMC Utrecht.
Provenance and peer review Not commissioned; externally peer reviewed.

Data sharing statement № additional data are available.

Open access This is an open access article distributed in accordance with the Creative Commons Attribution Non Commercial (CC BY-NC 4.0) license, which permits others to distribute, remix, adapt, build upon this work non-commercially, and license their derivative works on different terms, provided the original work is properly cited, appropriate credit is given, any changes made indicated, and the use is non-commercial. See: http://creativecommons.org/licenses/by-nc/4.0/

\section{REFERENCES}

1. Eslick GD, Coulshed DS, Talley NJ. The burden of illness of noncardiac chest pain. Aliment Pharmacol Ther 2002;16:1217-23.

2. Eslick GD, Jones MP, Talley NJ. Non-cardiac chest pain: prevalence, risk factors, impact and consulting--a population-based study. Aliment Pharmacol Ther 2003;17:1115-24.

3. Mourad G, Alwin J, Strömberg A, et al. Societal costs of non-cardiac chest pain compared with ischemic heart disease--a longitudinal study. BMC Health Serv Res 2013;13:403.

4. Ruddox V, Mathisen M, Otterstad JE. Prevalence and prognosis of non-specific chest pain among patients hospitalized for suspected acute coronary syndrome - a systematic literature search. BMC Med 2012;10:58

5. Ruigómez A, Massó-González EL, Johansson S, et al. Chest pain without established ischaemic heart disease in primary care patients: associated comorbidities and mortality. Br J Gen Pract 2009;59:e78-e86.

6. Leise MD, Locke GR, Dierkhising RA. Patients dismissed from the hospital with a diagnosis of noncardiac chest pain: cardiac outcomes and health care utilization. Mayo clinic proceedings. Elsevier, 2010.

7. Glombiewski JA, Rief W, Bösner S, et al. The course of nonspecific chest pain in primary care: symptom persistence and health care usage. Arch Intern Med 2010;170:251-5.

8. Roffi M, Patrono C, Collet JP, et al. 2015 ESC Guidelines for the management of acute coronary syndromes in patients presenting without persistent ST-segment elevation: Task Force for the Management of Acute Coronary Syndromes in Patients Presenting without Persistent ST-Segment Elevation of the European Society of Cardiology (ESC). Eur Heart J 2016;37:267-315.

9. Mol KA, Rahel BM, Meeder JG, et al. Delays in the treatment of patients with acute coronary syndrome: Focus on pre-hospital delays and non-ST-elevated myocardial infarction. Int $J$ Cardiol 2016;221:1061-6.

10. Webster R, Norman P, Goodacre S, et al. The prevalence and correlates of psychological outcomes in patients with acute non-cardiac chest pain: a systematic review. Emerg Med J 2012;29:267-73. 